\title{
Drogas ilícitas e lícitas e suas consequências durante a gestação: uma revisão da
}

\section{literatura}

\author{
Illicit and licit drugs and their consequences during pregnancy: a literature review \\ Drogas ilícitas y lícitas y sus consecuencias durante el embarazo: revisión de la literatura
}

Recebido: 04/10/2021 | Revisado: 10/10/2021 | Aceito: 19/10/2021 | Publicado: 20/10/2021

\author{
Ênale Augusta Silva Santana \\ ORCID: https://orcid.org/0000-0002-1607-5029 \\ Centro Universitário Santo Agostinho, Brasil \\ E-mail: anallesantanna@gmail.com \\ Yasmin de Sampaio Nunes \\ ORCID: https://orcid.org/0000-0002-2503-4616 \\ Centro Universitário Santo Agostinho, Brasil \\ E-mail: yasminsampaion@gmail.com \\ Daniela Fortes Neves Ibiapina \\ ORCID: https://orcid.org/0000-0002-2235-5545 \\ Centro Universitário Santo Agostinho, Brasil \\ E-mail: daniela.fortes@hotmail.com \\ Liejy Agnes dos Santos Raposo Landim \\ ORCID: https://orcid.org/0000-0002-8214-2832 \\ Centro Universitário Santo Agostinho, Brasil \\ E-mail: liejyagnes@gmail.com
}

\begin{abstract}
Resumo
Objetivo: analisar o consumo de drogas ilícitas e lícitas e suas consequências durante a gestação. Metodologia: Tratase de um artigo de revisão da literatura do tipo integrativa. a busca foi realizada nos bancos de dados da BVS, Scielo, Lilacs, PubMED e Google Acadêmico. Foram utilizados como DeCs (Descritores em Saúde) os termos: “Complicações na gravidez", "Drogas ilícitas" e "Gestação". foram inclusos artigos originais, indexados nas bases de dados selecionadas, nos idiomas: português e inglês; publicados entre os anos de 2016 e 2021. Os critérios de exclusão foram publicações de teses, anais de congressos e afins, dissertações, monografias e artigos que não tinham aderência à temática. Resultados: Constatou-se que entre as drogas lícitas mais consumidas estão o álcool e o tabaco, entre as ilícitas destaca-se a maconha, cocaína e o crack. Quanto as consequências do uso durante a gestação foram identificadas que o álcool e o tabaco são responsáveis por mal formação, síndrome alcoólica fetal, hipóxia fetal, baixo peso ao nascer, nascimento prematuro, problemas respiratórios, distúrbios neurológicos entre outros. Na mãe causa acidez gástrica, diminuição dos reflexos protetores respiratórios, descolamento da placenta, e parto prematuro. $\mathrm{O}$ crak e a cocaína ocasionam aborto, retardo do crescimento intrauterino, e problemas respiratórios, hipertensão, taquicardia e arritmia, infarto e até a morte. A maconha pode ocasionar anancefalia. Considerações finais: Mais estudos abordando a temática assim como oficinas e palestras, são necessários, com um direcionamento a conscientização das consequências do uso de drogas durante a gestação, o que trará resultados positivos.
\end{abstract}

Palavras-chave: Complicações na gravidez; Drogas ilícitas; Gestação.

\begin{abstract}
Objective: to analyze the consumption of illegal and legal drugs and their consequences during pregnancy. Methodology: This is an integrative literature review article. the search was carried out in the databases of the VHL, Scielo, Lilacs, PubMED and Academic Google. The terms "Pregnancy complications", "Illicit drugs" and "Pregnancy" were used as DeCs (Health Descriptors). original articles were included, indexed in the selected databases, in the languages: Portuguese and English; published between 2016 and 2021. Exclusion criteria were publications of theses, conference proceedings and the like, dissertations, monographs and articles that did not adhere to the theme. Results: It was found that among the most consumed legal drugs are alcohol and tobacco, among the illegal ones stand out marijuana, cocaine and crack. As for the consequences of use during pregnancy, it was identified that alcohol and tobacco are responsible for malformation, fetal alcohol syndrome, fetal hypoxia, low birth weight, premature birth, respiratory problems, neurological disorders, among others. In the mother, it causes gastric acidity, decreased respiratory protective reflexes, placental detachment, and premature birth. Crak and cocaine cause abortion, intrauterine growth retardation, and respiratory problems, hypertension, tachycardia and arrhythmia, heart attack and even death. Marijuana can lead to anancephaly. Final considerations: More studies addressing the topic, as well os workshops and lectures, are needed, with a focus on awareness of the consequences of drug use during pregnancy, which will bring positive results.
\end{abstract}

Keywords: Pregnancy complications; Illicit drugs; Gestation. 


\begin{abstract}
Resumen
Objetivo: analizar el consumo de drogas ilegales y legales y sus consecuencias durante el embarazo. Metodología: Este es un artículo de revisión integradora de la literatura. la búsqueda se realizó en las bases de datos de la BVS, Scielo, Lilacs, PubMED y Academic Google. Los términos "Complicaciones del embarazo", "Drogas ilícitas" y "Embarazo" se utilizaron como Descriptores de salud (DeC). Se incluyeron artículos originales, indexados en las bases de datos seleccionadas, en los idiomas: portugués e inglés; publicados entre 2016 y 2021. Los criterios de exclusión fueron publicaciones de tesis, actas de congresos y afines, disertaciones, monografías y artículos que no se ajustaran a la temática. Resultados: Se encontró que entre las drogas legales más consumidas se encuentran el alcohol y el tabaco, entre las ilegales destacan la marihuana, la cocaína y el crack. En cuanto a las consecuencias del consumo durante el embarazo, se identificó que el alcohol y el tabaco son responsables de malformaciones, síndrome alcohólico fetal, hipoxia fetal, bajo peso al nacer, parto prematuro, problemas respiratorios, trastornos neurológicos, entre otros. En la madre provoca acidez gástrica, disminución de los reflejos protectores respiratorios, desprendimiento de placenta y parto prematuro. El crak y la cocaína provocan aborto espontáneo, retraso del crecimiento intrauterino y problemas respiratorios, hipertensión, taquicardia y arritmia, infarto de miocardio e incluso la muerte. La marihuana puede provocar anancefalia. Consideraciones finales: Se necesitan más estudios que aborden el tema, así como talleres y conferencias, con un enfoque en la conciencia de las consecuencias del consumo de drogas durante el embarazo, lo que traerá resultados positivos.
\end{abstract}

Palabras clave: Complicaciones del embarazo; Drogas ilícitas; Gestación.

\title{
1. Introdução
}

A gravidez é um momento de grandes expectativas, surpresas e quase sempre muitas alegrias na vida da mulher. Laços se formam, caminhos e horizontes se abrem. Porém, nem sempre a gravidez termina como esperado, com um bebê saudável nos braços e uma mãe pronta para criá-lo em uma nova trajetória de vida (Carvalho, et al., 2019). As mudanças físicas e emocionais peculiares a esta situação afetarão a vida das mulheres e o ambiente social e familiar (Ventura, et al., 2020).

Outra fase importante da gravidez é o puerpério tempo de seis a oito semanas após o parto, que pode ser dividido em imediato (de 1 a 10 dias), tardio (11 a 45 dias) e remoto (a partir de 45 dias). Esse período inclui mudanças internas e externas, como desconforto nas mamas, sangramento vaginal, cicatrizes do parto, assim como inclui mudanças mentais (diminuição dos hormônios progesterona e estrogênio, e do aumento da prolactina, o hormônio que estimula a produção do leite materno) nas quais a mulher ainda necessita de cuidados e proteção. Nesse sentido, a mulher no puerpério precisa ser atendida em sua totalidade por meio de uma visão integral que leve em conta o contexto sociocultural e familiar (Andrade, et al., 2015).

O uso de drogas e álcool durante a gravidez é um dos fatores que influenciam negativamente essa trajetória, de forma extremamente impactante, o que a torna um grande problema de saúde pública, e um dos maiores problemas mundiais destacados pela imprensa e programas sociais, onde as drogas ilícitas ( álcool e o tabaco) são identificados como alguns fatores de exposição ambiental que podem levar ao desenvolvimento de algumas anormalidades fetais, como: anomalias faciais, defeitos de fechamento da parede abdominal, alterações do sistema nervoso central (SNC), sistema geniturinário e malformações cardíacas, etc. Portanto, se deve ressaltar também que muitos são os fatores que podem desencadear o uso de tais substâncias, dentre estes, problemas na família, estímulos sociais e da mídia, os quais, são registrados como possivelmente os responsáveis diretos (Carvalho, et al., 2019; Tacon, et al., 2020).

O número de mulheres usuárias de drogas tem aumentado e as diferenças de gênero no uso de drogas incluem fatores físicos, ambientais, sociais e de desenvolvimento. Esses fatores os tornam mais suscetíveis aos efeitos das drogas, causando maiores danos e aumentando as chances das mulheres de se tornarem dependentes (Ministério da cidadania, 2021).

No que diz respeito as usuárias de drogas, $90 \%$ estão em idade fértil, entre 15 e 40 anos, e $30 \%$ têm menos de 20 anos. Nesse âmbito, observa-se que $75,8 \%$ das gestantes iniciaram o pré-natal até a $16^{\mathrm{a}}$ semana de gestação e $73,1 \%$ realizaram o número mínimo de consultas até o parto. Isso se torna preocupante, pois fatores de risco como o uso de drogas por gestantes são identificados durante as consultas de pré-natal (Bianchini, et al., 2018).

É importante ressaltar que, durante a gravidez, quase todos as composições químicas passam pela placenta, sendo uma 
das funções a filtragem de substâncias presentes no sangue materno que podem ser prejudiciais ao feto. Porém, a placenta não filtra todos os elementos, é permeável a algumas substâncias importantes para o desenvolvimento embrionário, mas também a outras nocivas ao feto, incluindo vírus e drogas que o afetam diretamente, causando efeitos irreversíveis como má formação fetal e problemas neurológicos (Carvalho, et al., 2019).

Diante disso, entre as consequências de maior incidência estão a prematuridade e o baixo peso decorrentes do uso de drogas lícitas e ilícitas. O parto prematuro é definido como o nascimento do bebê com menos de trinta e sete semanas de gestação. O baixo peso ao nascer é definido como o peso inferior a 2500g. A cada ano, cerca de 20 milhões de bebês nascem em todo o mundo com baixo peso. Assim o parto prematuro e baixo peso ao nascer são considerados problemas em todo o mundo devido a sua associação com altas taxas de mortalidade e morbidade neonatal (Who, 2016).

Sendo assim o uso de drogas durante a gravidez é um problema de saúde pública em todo o mundo com terríveis consequências sociais como alto índice de morbidade e mortalidade infantil. Em gestantes, torna-se ainda mais importante, pois a exposição dessas pacientes às drogas pode levar ao comprometimento irreversíveis do binômio mãe-feto como parto prematuros, pré-eclâmpsia grave e aborto espontâneo (Bianchini, et al. 2018).

Diante disso, este estudo busca fazer uma revisão integrativa com o intuito de analisar o consumo de drogas ilícitas e lícitas e suas consequências durante a gestação.

\section{Metodologia}

Trata-se de um artigo de revisão da literatura do tipo integrativa, de natureza qualitativa e exploratória, com abordagem teórica, a revisão integrativa resulta da divisão em fases que auxiliam o processo metodológico de coleta de dados e análise de dados (Whittemore \& Knafl, 2005; Pompeo, et al. 2009). Trata-se de uma pesquisa que demonstra as consequências do consumo de drogas ilícitas e lícitas durante a gestação. Buscando incentivar que os leitores entendam os riscos do abuso de substâncias lícitas e ilícitas durante esse momento da vida, proporcionando um melhor aproveitamento do assunto e uma contribuição para que novos estudos sejam realizados.

A busca pelos artigos foi realizada em cinco bases de dados eletrônicos, da Biblioteca Virtual de Saúde da (BVS), e Scientific Electronic Library Online (Scielo), Literatura Latino Americana e do Caribe em Ciências da Saúde (Lilacs), National Library of Medicine (PubMED) e Google Acadêmico, para a verificação do estudo foram utilizados os descritores nos idiomas português "Complicações na gravidez", "Drogas ilícitas" e "Gestação" e no idioma inglês "Pregnancy complications ", “ Illicit drugs "and“ Pregnancy” cadastrados nos Descritores em Ciência da Saúde (DeCS).

Buscando aperfeiçoar o estudo as buscas foram realizadas entre os períodos de agosto de 2020 a maio de 2021, sendo selecionados inicialmente 52 artigos, e após a análise do objeto de estudo e os critérios de inclusão, permaneceram 10 artigos, sendo 8 na língua portuguesa e 2 na língua inglesa.

Para a seleção da amostra foram utilizados os seguintes critérios de inclusão: artigo original, indexado nas bases de dados selecionadas, nos idiomas: português e inglês; publicado entre os anos de 2016 a 2021. Os critérios de exclusão foram publicações de teses, anais de congressos e afins, dissertações, monografias e artigos que não tinham aderência à temática.

Em seguida, os estudos utilizados foram lidos e interpretados, o que levou à constituição do corpus, após leitura atenta da bibliografia, a literatura foi analisada sistematicamente e agrupada em um quadro, complementado por autor e ano de publicação, objetivo, amostra do estudo e os principais resultados.

\section{Resultados e Discussão}

No Quadro 1 estão reunidos os principais resultados sobre o consumo de drogas ilícitas e lícitas e suas consequências 
durante a gestação.

Quadro 1 - Distribuição dos resultados encontrados sobre os o consumo de drogas ilícitas e lícitas e suas consequências durante a gestação, publicados entre os períodos de 2016 a 2021 seguindo os autores, ano da publicação, objetivo, amostra da pesquisa e resultados.

\begin{tabular}{|c|c|c|c|c|}
\hline AUTOR/ANO & OBJETIVO & AMOSTRA & TIPO DE ESTUDO & RESULTADOS \\
\hline $\begin{array}{c}\text { Alexandrino, et. al. } \\
\text { (2016) }\end{array}$ & $\begin{array}{l}\text { Identificar as principais } \\
\text { malformações do sistema } \\
\text { nervoso fetal }(\mathrm{SN}) \\
\text { causadas pelo consumo do } \\
\text { álcool e tabagismo durante } \\
\text { a gravidez. }\end{array}$ & $\begin{array}{l}\text { artigos publicados em } \\
\text { periódicos indexados nas } \\
\text { bases de dados (SciELO, } \\
\text { MedLine, PubMed, } \\
\text { Lilacs). }\end{array}$ & $\begin{array}{ll}\text { revisão } & \text { não } \\
\text { sistemática. } & \end{array}$ & $\begin{array}{lrr}\text { Tanto o álcool } & \text { como o } \\
\text { tabaco } & \text { têm } & \text { ações } \\
\text { neurotóxicas } & \text { que podem } \\
\text { levar a } & \text { alterações } \\
\text { morfológicas } & & \text { e/ou } \\
\text { funcionais do } & \mathrm{SN} \text { fetal. }\end{array}$ \\
\hline $\begin{array}{l}\text { Maia; Pereira \& } \\
\text { Meneses } \\
\text { (2016) }\end{array}$ & $\begin{array}{l}\text { Descrever as } \\
\text { consequências do uso de } \\
\text { drogas durante a gravidez. }\end{array}$ & $\begin{array}{l}\text { A amostra foi composta } \\
\text { por } 100 \text { usuárias grávidas }\end{array}$ & $\begin{array}{l}\text { Estudo observacional } \\
\text { e quantitativo com } \\
\text { abordagem descritiva. }\end{array}$ & $\begin{array}{l}\text { Concluímos que }(37 ; \\
2,05 \%) \text { das grávidas } \\
\text { entrevistadas faziam uso de } \\
\text { etanol, }(19 ; 1,00 \%) \text { eram } \\
\text { fumantes, }(22 ; 1,22 \%) \text { eram } \\
\text { usuárias da maconha, (17; } \\
0,94 \%) \text { faziam uso de } \\
\text { cocaína e }(47 ; 2,61 \%) \text { eram } \\
\text { usuárias de crack, } \\
\text { colocando em risco o } \\
\text { desenvolvimento do feto e } \\
\text { de sua saúde. }\end{array}$ \\
\hline $\begin{array}{l}\text { Pena, et. al. } \\
\text { (2017) }\end{array}$ & $\begin{array}{l}\text { Verificar a influência do } \\
\text { uso de álcool e tabaco no } \\
\text { peso do bebê ao nascer. }\end{array}$ & $\begin{array}{l}\text { A amostra foi constituída } \\
\text { por } 300 \text { prontuários. }\end{array}$ & $\begin{array}{l}\text { Estudo exploratório, } \\
\text { descritivo, } \\
\text { retrospectivo, } \\
\text { documental. }\end{array}$ & $\begin{array}{l}\text { O uso de tabaco e do álcool } \\
\text { durante a gestação interfere } \\
\text { negativamente no peso do } \\
\text { recém-nascido ao nascer. }\end{array}$ \\
\hline $\begin{array}{l}\text { Santos \& Gavioli, } \\
\text { (2017) }\end{array}$ & $\begin{array}{l}\text { Avaliar o risco relacionado } \\
\text { ao consumo de drogas } \\
\text { entre gestantes. }\end{array}$ & 209 gestantes & estudo transversal. & $\begin{array}{l}\text { dentre as gestantes, } 18,1 \% \\
\text { apresentaram nível de risco } \\
\text { moderado e elevado } \\
\text { relacionado ao consumo de } \\
\text { tabaco, } 27,2 \% \text { de álcool e } \\
1,9 \% \text { de maconha. }\end{array}$ \\
\hline Pereira, et. al. (2018) & $\begin{array}{l}\text { Avaliar a relação entre o } \\
\text { uso de substâncias } \\
\text { psicoativas na gestação e a } \\
\text { ocorrência de morbidade } \\
\text { materna grave (MMG), } \\
\text { resultados perinatais e } \\
\text { repercussões } \\
\text { desenvolvimento } \\
\text { neuropsicomotor } \\
\text { crianças expostas. }\end{array}$ & $\begin{array}{l}\text { Mulheres com MMG } \\
\text { foram consideradas casos. } \\
\text { Controles foram mulheres } \\
\text { com gestação de baixo } \\
\text { risco, admitidas no } \\
\text { mesmo período que os } \\
\text { casos. }\end{array}$ & caso-controle & $\begin{array}{l}\text { A prevalência do uso de } \\
\text { drogas lícitas ou ilícitas } \\
\text { durante a gravidez foi de } \\
\text { aproximadamente } 17 \% \text {. } \\
\text { Entre os usuários de drogas, } \\
63,9 \% \text { usavam álcool, } \\
58,3 \% \text { fumo, } 9,2 \% \text { cocaína / } \\
\text { crack e } 4,6 \% \text { maconha. Não } \\
\text { houve associação entre uso } \\
\text { de drogas durante a } \\
\text { gravidez e SMM, embora o } \\
\text { uso de tabaco durante a } \\
\text { gravidez tenha sido } \\
\text { associado a sangramento, } \\
\text { presença de critérios } \\
\text { clínicos de near miss } \\
\text { (NMCC) e alteração no } \\
\text { desenvolvimento infantil; o } \\
\text { uso de álcool foi associado } \\
\text { à asfixia neonatal; e o uso } \\
\text { de cocaína / crack foi } \\
\text { associado à ocorrência de } \\
\text { algumas complicações } \\
\text { clínicas durante a gravidez. }\end{array}$ \\
\hline
\end{tabular}




\begin{tabular}{|c|c|c|c|c|}
\hline $\begin{array}{l}\text { Carvalho et al. } \\
\text { (2019) }\end{array}$ & $\begin{array}{l}\text { Relacionar o uso de álcool } \\
\text { e outras drogas durante a } \\
\text { gestação à RCF em } \\
\text { puérperas cadastradas na } \\
\text { Estratégia de Saúde da } \\
\text { Família } \\
\text { município de Araguari- } \\
\text { MG. }\end{array}$ & 70 puérperas & $\begin{array}{l}\text { estudo de corte } \\
\text { transversal }\end{array}$ & $\begin{array}{l}25,70 \% \text { das puérperas } \\
\text { afirmaram ter utilizado } \\
\text { algum tipo de droga lícita } \\
\text { ou ilícita durante a } \\
\text { gestação, apenas o uso de } \\
\text { crack/cocaína apresentou } \\
\text { relação estatisticamente } \\
\text { significativa com a a } \\
\text { prevalência de recém- } \\
\text { nascidos (RN) pequenos } \\
\text { para a idade gestacional e } \\
\text { com baixo peso ao nascer. }\end{array}$ \\
\hline $\begin{array}{l}\text { Maia et al. } \\
\quad(2019)\end{array}$ & $\begin{array}{l}\text { Identificar as drogas mais } \\
\text { utilizadas por gestantes. }\end{array}$ & 30 gestantes & $\begin{array}{l}\text { Estudo transversal de } \\
\text { caráter observacional, } \\
\text { descritivo e } \\
\text { exploratório }\end{array}$ & $\begin{array}{lrr}\begin{array}{l}\text { Durante } \\
\text { gestacional: }\end{array} & 60 \% \text { período } \\
\text { mulheres } & \text { consumiam } \\
\text { álcool, } 30 \% & \text { usavam } \\
\text { cigarro, 6,7\% } & \text { utilizavam } \\
\text { álcool e cigarro } & \text { associados } \\
\text { e } 3,3 \% \text { das } & \text { gestantes } \\
\text { usavam concomitantemente, } \\
\text { álcool, cigarro, maconha, } \\
\text { cocaína e crack. }\end{array}$ \\
\hline $\begin{array}{l}\text { Grant et al. } \\
\quad(2020)\end{array}$ & $\begin{array}{l}\text { Atualizar os dados sobre as } \\
\text { consequências do uso da } \\
\text { maconha no } \\
\text { desenvolvimento durante a } \\
\text { gravidez e a lactação. }\end{array}$ & 20 artigos & Revisão sistemática & $\begin{array}{lr}\text { Foram } & \text { evidenciados } \\
\text { impactos negativos nas } \\
\text { habilidades cognitivas } \\
\text { especificas, principalmente } \\
\text { na memória. A longo prazo } \\
\text { há impactos na saúde } \\
\begin{array}{l}\text { psicológica, como } \\
\text { depressão, ansiedade e } \\
\text { delinquência. }\end{array}\end{array}$ \\
\hline $\begin{array}{l}\text { Silva et al. } \\
\quad(2020)\end{array}$ & $\begin{array}{l}\text { Estimar a prevalência do } \\
\text { uso de drogas de abuso nas } \\
\text { gestantes e associar com as } \\
\text { variáveis escolaridade, } \\
\text { renda familiar, raça e } \\
\text { número de gestações. }\end{array}$ & 114 gestantes & $\begin{array}{l}\text { pesquisa descritiva, } \\
\text { transversal, } \\
\text { quantitativa. }\end{array}$ & $\begin{array}{l}\text { A droga de abuso mais } \\
\text { utilizada foi o álcool, } \\
\text { seguido de tabaco. O uso } \\
\text { concomitante } \\
\text { entre as drogas apresentou- } \\
\text { se significativo sendo } \\
\text { álcool/tabaco e drogas } \\
\text { ilícitas/tabaco. }\end{array}$ \\
\hline $\begin{array}{l}\text { Lopes et al. } \\
\text { (2021) }\end{array}$ & $\begin{array}{l}\text { Investigar a prevalência do } \\
\text { uso de substâncias } \\
\text { psicoativas em gestantes e } \\
\text { puérperas atendidas no } \\
\text { ambulatório de um } \\
\text { Hospital Escola. }\end{array}$ & 174 gestantes e puérperas. & $\begin{array}{l}\text { pesquisa transversal, } \\
\text { descritiva }\end{array}$ & \begin{tabular}{lrr} 
O tabaco & \multicolumn{2}{r}{ constitui a } \\
substância com maior \\
frequência de uso; que \\
desencadeia & maior \\
desejo/urgência & de \\
consumo; maior taxa de \\
tentativas de diminuição.
\end{tabular} \\
\hline
\end{tabular}

Fonte: Dados da pesquisa (2021).

É importante observar no quadro 01 a distribuição desses resultados que consistem e se delimitam a um único objetivo, que é analisar o consumo de drogas ilícitas e lícitas e suas consequências durante a gestação. A gravidez é um momento especial na vida da mulher e de sua família, que exige cuidados com seus hábitos e estilo de vida, por exemplo, o uso de drogas lícitas e ilícitas que podem afetar a saúde da mãe e do feto.

Partindo dessa análise Alexandrino et. al., (2016) apontaram as principais malformações do sistema nervoso fetal (SNF) causadas pelo consumo de álcool e tabaco durante a gravidez, ressaltando que tanto o álcool quanto o tabaco apresentam efeitos neurotóxicos que podem levar a alterações morfológicas e/ou funcionais do sistema nervoso. O álcool é identificado como o teratógeno mais comum em humanos, e o tabaco se distingue por seu consumo ativo e passivo por mulheres grávidas. A exposição do feto ao álcool induz alterações que levam à neuronegeneração de neurônios dopaminérgicos, gânglios da base, 
giro denteado do hipocampo, corpo caloso e cerebelo. A nicotina do cigarro atinge níveis elevados no feto e interfere diretamente na neurulação, causando defeitos do tubo neural, espinha bífida, exencefalia e outros efeitos funcionais. As malformações causadas pelo álcool e pelo fumo são as principais causas evitáveis de teratógenos em humanos.

Maia; Pereira e Meneses (2016) Descreveram as consequências do uso de drogas durante a gravidez, concluindo que o uso de drogas durante a gravidez pode trazer sérias consequências tanto para as mães como para os recém nascidos, sendo imprescindível que o diagnóstico desse problema seja realizado durante as anamneses e consultas de planejamento familiar e Pré-natal. Em relação as substâncias mais consumidas o crack foi o que apresentou a maior proporção de usuárias, seguido do consumo do etanol, da maconha, cigarro normal e da cocaína, tais números parecem ser pequenos, mas em relação à população estudada, representam um grande número de mulheres que relataram o uso de alguma droga durante a gravidez.

Pena et. al., (2017) verificaram a prevalência de etilismo e tabagismo em gestantes assim como verificaram a influência do uso de álcool e tabaco no peso do bebê ao nascer, evidenciando que há uma grande prevalência de tabagismo e etilismo entre as mulheres durante a gestação e a interferência dessa prática no peso do bebê ao nascer. A frequência do consumo de álcool durante a gravidez foi inferior à do tabaco na amostra estudada. Não houve diferença estatisticamente significativa entre o peso ao nascer de filhos de mães alcoólatras ou não, talvez porque o número de mulheres que confirmaram esse hábito durante a gravidez tenha sido muito pequeno. Houve diferença estatisticamente significativa no peso dos recémnascidos após o nascimento em relação às mães fumantes, o que permite concluir que o tabagismo durante a gravidez afeta negativamente o ganho de peso do bebê.

Santos e Gavioli (2017) avaliaram o risco relacionado ao consumo de drogas entre gestantes, concluindo que a maioria das gestantes estavam em baixo risco relacionado ao uso de drogas como o álcool e o tabaco. Em relação à faixa de risco para distúrbios de saúde relacionados ao consumo, ou seja, os riscos classificados como moderado e alto, o álcool apresentou um maior índice em relação ao tabaco. Em relação ao uso de drogas ilícitas, as mulheres grávidas estavam na faixa de baixo risco para o consumo de maconha a qual apresentou uma porcentagem bem pequena de risco moderado. Não havia mulheres grávidas em risco relacionado ao uso de cocaína. Tabaco e álcool foram as principais drogas de abuso legal usado por mulheres grávidas. Maconha era a principal droga ilícita consumida. O consumo de álcool, tabaco e maconha foram de moderado a altos níveis de risco, sendo significativamente maiores nesta amostra quando comparado aos resultados de outros estudos.

Nesse contexto Pereira et. al., (2018) avaliaram a relação entre o uso de substâncias psicoativas na gestação e a ocorrência de morbidade materna grave (MMG), concluindo que a droga mais consumida entre as gestantes foi o álcool, o tabaco, seguidas da cocaína, crack e maconha, e como consequências desse consumo foi associado a hemorragia, presença de critérios clínicos de near-miss e alteração no desenvolvimento infantil. O uso do álcool foi associado a asfixia neonatal, já o uso de cocaína e crack a ocorrência de alguma complicação clínica na gestação. Quanto ao desfecho em recém-nascidos, o uso de drogas não se associou à prematuridade. No entanto, o risco de apresentar índice de Apgar $<7$ em recém-nascidos foi de 2,3 vezes maiores. $\mathrm{O}$ consumo de álcool representou a maior ameaça para esse resultado. $\mathrm{O}$ uso de tabaco e cocaína foi associado a um atraso no desenvolvimento infantil na escala de Denver de 6 meses a 5 anos após o parto. Desta forma o uso de drogas não mostrou ser um fator associado a MMG.

Carvalho et al., (2019) constataram que pelo menos 25,70\% das puérperas utilizaram alguma substância durante a gestação, dentre estas destacam-se o álcool e o tabaco, seguido do crack e da cocaína. Em relação ao consumo de álcool, constatou-se que a prática não se associou às mudanças na idade gestacional, peso corporal para idade gestacional e peso ao nascer. Analisando a influência do tabagismo nas condições do parto, constatou-se que essa prática não afetou a idade gestacional, o peso para a idade gestacional e o peso isolado do recém-nascido. Ao observar os efeitos do crack e da cocaína nas condições de trabalho, foi possível verificar que essa prática não esteve relacionada às mudanças na idade gestacional, mas 
foi possível verificar que houve relação estatisticamente significativa entre o uso dessas drogas ilícitas e o padrão de peso corporal para a idade gestacional, aumentando a incidência de recém-nascidos pequenos em relação à idade gestacional, bem como o que ocorre com o peso do recém-nascido ao nascer, aumentando a incidência de baixo peso ao nascer.

Maia, et al. (2019) Identificaram as drogas mais utilizadas por gestantes, evidenciando que entre as substâncias psicoativas mais citadas pelas gestantes do estudo está o álcool, seguido do cigarro e o uso combinado do álcool e cigarro. Em relação a classe, as drogas lícitas demonstram serem as mais utilizadas, o que se justifica talvez pelo fácil acesso e baixo custo e por serem socialmente aceitas. Desta forma, ressalta-se a importância dos profissionais de saúde, especificamente nas consultas de pré natal e atenção primária a passarem por um aprimoramento assistencial a respeito do uso de drogas na gestação a fim de prevenir e detectar a utilização de tais substâncias.

De acordo com os resultados encontrados por Grant et al. (2020) os estudos não encontraram uma assinatura fenotípica única para a exposição pré-natal, mas foi relatado um risco aumentado de defeitos congênitos, particularmente gastrosquise. Mudanças no crescimento fetal foram descritas em alguns estudos epidemiológicos, mas os padrões de crescimento físico de longo prazo não parecem ser afetados. A exposição pré-natal à cannabis geralmente não está associada a uma redução no QI global, mas pode afetar negativamente certas habilidades cognitivas, especialmente atenção e memória. Os efeitos de longo prazo sobre a saúde mental incluem taxas aumentadas de sintomas de depressão e ansiedade, bem como crimes. Relativamente pouco se sabe sobre o risco do uso materno de cannabis durante a lactação, mas os dados sugerem que a exposição na infância é relativamente baixa em comparação com a exposição materna.

Silva et al., (2020) identificaram uma alta prevalência do uso de drogas de abuso nas gestantes, assim como o uso de drogas ilícitas, predominando o uso do álcool e do tabaco, quanto aos fatores socioeconômicos e culturais, foram observados baixo nível de escolaridade, predomínio da raça não branca renda familiar de 1 a 2 salários mínimos, e as mulheres eram multíparas. o que indica um problema de saúde pública, desde que essas gestantes são consideradas de alto risco. Compreendese então que o uso de drogas atua conjuntamente com aspectos sociais, sendo necessária a realização de um plano de cuidado individualizado e eficaz, afim de garantir a prevenção a saúde da mulher e do seu bebê.

Lopes et al., (2021) constatam em seu estudo que as substâncias mais consumidas foram as derivadas do tabaco, sendo necessário ressaltar que essas substancias são as que mais despertam o desejo ou a urgência do consumo. Além disso, representam os maiores números de tentativa de redução ou abstinência, onde as gestantes apresentaram índices de dependência moderada a grave. Desta forma, entende se que a avaliação do uso de substâncias psicoativas em gestantes e puérperas, é imprescindível por se tratar de uma população singular, cujas características sociodemográficas se somam às características obstétricas e ginecológicas, necessitando de maior atenção dos profissionais de saúde devido aos reflexos que impõe sobre a saúde da mãe e do bebê.

\section{Considerações Finais}

Foi possível inferir que entre as drogas lícitas mais consumidas o álcool se destaca, já as ilícitas a maconha foi a mais citada, em relação as consequências mais relatadas destacam-se a má-formação e o baixo peso ao nascer. Pode-se constatar através dos resultados que os prejuízos causados à saúde da gestante e do feto são imensuráveis, repercutindo diretamente no decorrer de suas vidas. Dessa forma esse estudo se demonstra de grande relevância desde que apresenta resultados que poderão auxiliar no processo de tratamento e prevenção para que sejam evitados o uso de drogas ilícitas e lícitas durante a gestação. Sendo assim mais estudos abordando a temática assim como oficinas e palestras, são necessários, com um direcionamento a conscientização das consequências do uso de drogas durante a gestação, sendo imprescindível o enfoque nas políticas públicas que enfatizem a importância da prevenção e do tratamento, o que trará resultados positivos.

Por fim, ressalta-se a importância do desenvolvimento de novas pesquisas envolvendo a temática drogas e gestação, 
tendo em vista que esse problema vem acometendo milhões de mulheres em todo o mundo, o que irá contribuir diretamente para a literatura científica sobre o tema apresentado, assim como para pesquisadores e profissionais da área.

\section{Referências}

Abraham, C. F., \& Hess, A. R. B. (2016). Efeitos do uso do Crack Sobre o feto e o Recém-nascido: Um Estudo de Revisão. Revista de Psicologia da IMED, $8(1): 38-51$.

Andrade, R. D., et al. (2015). Fatores relacionados à saúde da mulher no puerpério e repercussões na saúde da criança. Esc. Anna Nery Rev. Enferm, 19(1):181-6.

Alexandrino, J. S., et al. (2016). Repercussões neurológicas nos fetos expostos a drogas lícitas durante a gestação: uma reflexão teórica SANARE, Sobral. 15(1), 82-9.

Bianchini, B. V., et al. (2018). Uso de drogas lícitas e ilícitas na gestação e as repercussões no nascimento prematuro e de baixo peso. Disciplinarum Scientia. Série: Ciências da Saúde, Santa Maria. 19(3), 611-22.

Carvalho, E. N., et al. (2019). A restrição do crescimento fetal como consequência do consumo de álcool e outras drogas na gestação: um estudo transversal. Revista Interdisciplinar Ciências Médicas, 4(1): 44-49.

Grandt, K. S., et al. (2020). Update on the developmental consequences of cannabis use during pregnancy and lactation. Birth Defects Research. 112 (15):1126-1138.

Lopes, K. B., et al. (2021). Prevalência do uso de substâncias psicoativas em gestantes e puérperas. REUFSM. Santa Maria, 11(45): 1-19.

Maia, J. A, Pereira, L. A., \& Menezes, F. A. (2016). Consequências do uso de drogas durante a gravidez. Revista Enfermagem Contemporânea, 4(2). 1-8.

Maia, J. A., et al. (2019). Uso de drogas por mulheres durante o período gestacional. Revista Enfermagem Contemporânea, 8(1): 25-32.

Ministério da cidadania. (2021). Conhecendo os efeitos do uso de drogas na gestação e as consequências para os bebês. Brasília: Ministério da Cidadania.

Pompeo, D. A., et al. (2009). Revisão integrativa: etapa inicial do processo de validação de diagnóstico de enfermagem. Acta Paul Enferm, 22(4):434-8.

Pena, et al. (2017). Uso do álcool e tabaco na gestação: influência no peso do recém-nascido. Revista saúde, 11(1-2): 74-82.

Pereira, et al. (2018). Drug Use during Pregnancy and its Consequences: Uso de drogas na gestação e suas consequências: um estudo de caso-controle aninhado sobre morbidade materna grave. Rev. Bras Ginecol Obstet, (40):518-526.

Santos, R. M. S., \& Gavioli, A. A. (2017). Risco relacionado ao consumo de drogas de abuso em gestantes. Rev Rene, 18(1):35-42.

Silva, F. T. R., et al. (2020) Prevalência e fatores associados ao uso de drogas de abuso por gestantes. Rev. Bras. Saúde Mater. Infant., Recife. 20(4): 11091115 .

Tacon, F. S. D., et al. (2020). Drogas e gravidez: efeitos na morfologia fetal. Research, Society and Development, 9(7): 1-17.

Ventura, J. (2020). Estigma associado a gestante/puérpera usuária de crack: ameaça que representa a instituição. Research, Society and Development, 9(2): 117.

Whittemore, R. \& Knafl, K. (2005). The integrative review: updated methodology. J Adv Nurs, 52(5):546-53.

Who - World Health Organization. (2016). Preterm birth. 18 maio 2021. https://bitly/2WKJsyI.

Wong, S., et al. (2011). Substance Use in Pregnancy. Journal of Obstetrics and Gynaecology, Canada. 33 (4):367-384. 